\title{
On the conjunctive capacity of graphs
}

\author{
Miroslav Chlebík ${ }^{1}$ and Janka Chlebíková ${ }^{2}$ \\ 1 University of Sussex, UK, m.chlebik@sussex.ac.uk \\ 2 University of Portsmouth, UK, janka.chlebikova@port.ac.uk
}

\begin{abstract}
The investigation of the asymptotic behaviour of various graph parameters in powers of a fixed graph $G=(V, E)$ is motivated by problems in information theory and extremal combinatorics. Considering various parameters and/or various notions of graph powers we can arrive at different notions of graph capacities, of which the Shannon capacity is best known. Here we study a related notion of the so-called conjunctive capacity of a graph $G, C_{\mathrm{AND}}(G)$, introduced and studied by Gargano, Körner and Vaccaro [5], [6]. To determine $C_{\mathrm{AND}}(G)$ is a convex programming problem. In this paper we show that the optimal solution to this problem is unique and describe the structure of the solution in any (simple) graph. We show that its reciprocal value $v c_{C}(G):=\frac{1}{C_{\mathrm{AND}}(G)}$ is an optimal solution of the newly introduced problem of Minimum Capacitary Vertex Cover that is closely related to the LP-relaxation of the Minimum Vertex Cover Problem. We also describe its close connection with the binding number/binding set of a graph, and with the strong crown decomposition of graphs introduced in [2].
\end{abstract}

\section{Introduction}

An induced complete subgraph of a graph $G$ is called a clique and the cardinality of the largest clique of $G$ is called its clique number, $\omega(G)$. The analysis of its growth in large product graphs leads to several interesting problems in combinatorics. The problem was originated by Shannon [11] in 1956 in his analysis of the capability of certain noisy communication channels to transmit information in an error-free manner. Shannon's model associated a graph with every channel. In our notation the vertex set of the graph represents the symbols that can be transmitted through the channel and two vertices are connected by an edge if the corresponding symbols can never get confused by the receiver. This model naturally leads to a product of graphs through the repeated use of the channel for the transmission of symbol sequences of some fixed length $n$. If the graph $G=(V, E)$ is a simple graph (all graphs in this paper are assumed simple), then $G^{n}$ denotes the graph with vertex set $V^{n}$ whose edge set contains those pairs of sequences in $V^{n}$ which can never get confused by the receiver. Formally, $\{x, y\} \in E\left(G^{n}\right)$ if and only if $\exists i\left\{x_{i}, y_{i}\right\} \in E$, where $E$ denotes the edge set of the graph $G$ and $x=\left(x_{1}, x_{2}, \ldots, x_{n}\right), y=\left(y_{1}, y_{2}, \ldots, y_{n}\right)$ are elements of $V^{n}$. Following Berge [1], $G^{n}$ is called the $n$-th co-normal power of $G$. Shannon [11] observed that if $K$ is a clique in $G$ then $K^{n}$ is a clique in $G^{n}$, whence the size 
of the clique number of $G^{n}$ is at least the $n$-th power of the clique number of $G$. (In fact, these two quantities coincide whenever the clique number of $G$ equals its chromatic number. This observation led Berge to his celebrated concept of perfect graphs.) The observation logically leads to the concept introduced by Shannon - to determine the always existing limit $C(G)=\lim _{n \rightarrow \infty} \frac{\log \omega\left(G^{n}\right)}{n}$, which is called the Shannon capacity of $G$. An analogous approach was initiated in [9] where the notion of Sperner capacity was introduced as the natural counterpart of Shannon capacity in the case of directed graphs. These notions became the key to the solution of some important open problems in extremal combinatorics [5], [6].

We have to warn that many traditional papers use a complementary language and define Shannon capacity $C(G)$ as our $C(\bar{G})$, when $C(G)$ is then defined using independent sets in the normal powers instead of cliques in the co-normal powers of $G$.

One can also extend the definition of Shannon capacity to graph families. Let $\mathcal{G}=\left\{G_{1}, G_{2}, \ldots, G_{k}\right\}$ be a family of graphs on a common set of vertices $V\left(G_{i}\right)=V$. We define the Shannon capacity $C(\mathcal{G})$ of a family $\mathcal{G}$ by

$$
C(\mathcal{G})=\lim _{n \rightarrow \infty} \frac{1}{n} \log \omega\left(G_{1}^{n} \cap G_{2}^{n} \cap \cdots \cap G_{k}^{n}\right) .
$$

The Shannon capacity of a family is motivated as the zero-error capacity of the compound channel described by $\mathcal{G}$. Additional motivation comes from extremal combinatorics.

Let us be given a graph $G=(V, E)$. The Shannon capacity can be considered an "OR-capacity" for $G$. In its definition, two elements $x$ and $y$ of $V^{n}$ are considered "really different" if there is a coordinate $i$ for which $\left\{x_{i}, y_{i}\right\} \in E$, i.e. at least one of the edges of $G$ occurs among the coordinate pairs $\left\{x_{i}, y_{i}\right\}$. The important fact is that it can be either one of the edges of $G$ - this is what we mean by calling $C(G)$ above as OR-capacity.

The second natural capacity associated with the graph $G$ is "AND-capacity" (the conjunctive capacity), denoted by $C_{\mathrm{AND}}(G)$. In its definition one would require that every edge of the graph $G$ be present among the coordinate pairs of the sequences. More explicitly, now we define the $n$-th conjunctive power of $G$ as the graph $G_{\mathrm{AND}}^{n}=\left(V^{n}, E\left(G_{\mathrm{AND}}^{n}\right)\right)$ such that $\left\{\left(x_{1}, x_{2}, \ldots, x_{n}\right),\left(y_{1}, y_{2}, \ldots, y_{n}\right)\right\} \in$ $E\left(G_{\mathrm{AND}}^{n}\right)$ if and only if for every $e \in E$ there exists a coordinate $1 \leq i \leq n$ such that $\left\{x_{i}, y_{i}\right\}=e$, and $C_{\mathrm{AND}}(G)=\lim _{n \rightarrow \infty} \frac{\log \omega\left(G_{\mathrm{AND}}^{n}\right)}{n}$.

The conjunctive capacity is clearly a special case of the Shannon capacity of a family of graphs. Given a graph $G=(V, E)$, let us denote by $\mathcal{G}:=\mathcal{F}(G)$ the family of single-edge graphs obtained by considering for every $e \in E$ the graph $G_{e}$ defined by setting $V\left(G_{e}\right)=V$ and $E\left(G_{e}\right)=\{e\}$. Thus $\mathcal{G}:=\mathcal{F}(G)$ is the family of the $|E|$ different single-edge graphs and the conjunctive capacity, $C_{\mathrm{AND}}(G)$, of $G$ is just the Shannon capacity $C(\mathcal{F}(G))$ of this simple family of graphs.

The problem of determining the Shannon capacity $C(G)$ of a given graph $G$ is not even known to be NP-hard although it seems plausible that it is in fact 
much harder; the problem of deciding whether the Shannon capacity of a given graph exceeds a given value is not known to be decidable. On the other hand, to determine the conjunctive capacity $C_{\mathrm{AND}}(G)$ is algorithmically much simpler.

In this paper we show that the reciprocal value $\frac{1}{C_{\mathrm{AND}}(G)}$ is an optimal solution of the newly introduced problem of Minimum Capacitary Vertex Cover that is closely related to the LP-relaxation of the Minimum Vertex Cover Problem (Section 2). We prove that the optimal solution to the problem is unique (Section 5) and describe the structure of the solution in any (simple) graph (Section 6). We also point out its close connection with the binding number/binding set of a graph and the strong crown decompositions of graphs described in [2].

\section{The conjunctive capacity}

In [3] and [5] the authors study the asymptotic value $C_{\mathrm{AND}}(G)$. These results have been used to answer a long-standing open question on the asymptotics of the maximum number of qualitatively independent partitions in the sense of Rényi [10]. They provide a computable formula for determining the conjunctive capacity $C_{\mathrm{AND}}(G)$ of any graph $G=(V, E)$ as

$$
C_{\mathrm{AND}}(G)=\max _{P} \min _{\{u, v\} \in E}(P(u)+P(v)) h\left(\frac{P(u)}{P(u)+P(v)}\right),
$$

where the maximum is over all probability distributions $P$ on the vertex set $V$ of $G, h$ is the binary entropy function, $h(t)=-t \log t-(1-t) \log (1-t), \quad t \in(0,1)$ which is continuously extended by $h(0)=h(1)=0$ to the interval $[0,1]$. (Here and in the sequel logarithms are to the base 2.) The formula (1) is starting point to our results.

In this paper we study the structure of the optimal solution of the convex programming problem defined on the graph $G$, introduced by the right hand side of (1). In what follows we use the function $f:[0, \infty) \times[0, \infty) \rightarrow[0, \infty)$ defined by $f(x, y)=(x+y) h\left(\frac{x}{x+y}\right), \quad(x, y) \in[0, \infty) \times[0, \infty) \backslash\{(0,0)\}$, and $f(0,0)=0$. Notice that $f$ is continuous on $[0, \infty) \times[0, \infty)$, and it simplifies to $f(x, y)=(x+y) \log (x+y)-x \log x-y \log y$ for $x, y>0$.

Let $G=(V, E)$ be a graph. The conjunctive capacity of $G$ can then be expressed as

$$
C_{\mathrm{AND}}(G)=\max _{P} \min _{\{u, v\} \in E} f(P(u), P(v))
$$

where maximum is taken over all probability distributions $P$ on $V$. (If $G$ does not have any edges then we set $C_{\mathrm{AND}}(G)=$ infinity.) If $G$ has at least one edge, a distribution $P$ for which the maximum in the definition of $C_{\mathrm{AND}}(G)$ is achieved clearly exists. We will show later that such a distribution is unique and describe the structure of this optimal distribution. 


\section{The Minimum Capacitary Vertex Cover problem}

Recall that in the LP-relaxation of Minimum Vertex Cover the task is for a given graph $G=(V, E)$ to minimize its fractional vertex cover

$$
v c_{*}(G)=\min _{x}\{x(V):(x(u), x(v)) \in T \text { for every }\{u, v\} \in E\},
$$

where $T=\{(a, b) \in[0, \infty) \times[0, \infty): a+b \geq 1\}$ and the minimum is taken over all $x: V \rightarrow[0, \infty)$. (Here and in the sequel $x(V):=\sum_{u \in V} x(u)$.)

Now let us consider for a graph $G=(V, E)$ and any fixed $t>0$ the following minimization problem over all nonnegative functions $x: V \rightarrow[0, \infty)$ :

$$
g(t)=\min _{x}\{x(V): f(x(u), x(v)) \geq t \text { for every }\{u, v\} \in E\} .
$$

The edge constraints in (4) are similar to those of the LP-relaxation of the Minimum Vertex Cover problem for $G$. If we denote

$$
T(t)=\{(a, b) \in[0, \infty) \times[0, \infty): f(a, b) \geq t\},
$$

then (4) reads as $g(t)=\min _{x}\{x(V):(x(u), x(v)) \in T(t) \quad$ for every $\{u, v\} \in E\}$.

It can easily be verified by direct computation that the function $f$ is positive homogene which means $f(c x, c y)=c f(x, y)$ for each $c>0$ and each $(x, y) \in$ $[0, \infty) \times[0, \infty)$. So the function $g(t)$ has nice scaling properties, in particular $g(t)=t g(1)$ for every $t>0$. Hence $C_{\mathrm{AND}}(G)$ is the only $t>0$ such that $g(t)=1$ and consequently, $g(1)=\frac{1}{C_{\mathrm{AND}}(G)}$ as $\left.g\left(C_{\mathrm{AND}}(G)\right)=1\right)$.

To determine $C_{\mathrm{AND}}(G)$ and a maximizing distribution $P$ for $G$ in (2) we introduce the following optimization problem that we call the Minimum Capacitary Vertex Cover problem:

Instance: A graph $G=(V, E)$.

Feasible solution: A function $x: V \rightarrow[0,+\infty)$ satisfying constraints $(x(u), x(v)) \in$ $T(1)$ for every edge $\{u, v\} \in E$, where $T(1)$ is defined by $T(1)=\{(a, b) \in[0, \infty) \times[0, \infty): f(a, b) \geq 1\}$.

Goal: To minimize $x(V):=\sum_{u \in V} x(u)$ over all feasible solutions.

Denote $v c_{C}(G)$ the optimum value of the Minimum Capacitary Vertex Cover problem.

One can compare the shapes of $T$ and $T(1)$ (Figure 1):

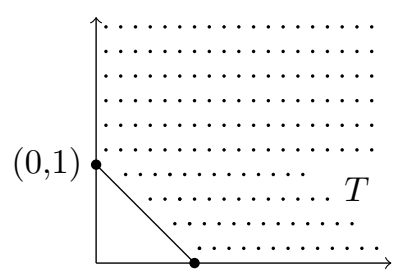

$(1,0)$

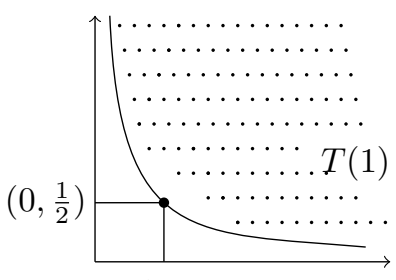

$\left(\frac{1}{2}, 0\right)$

The main difference is in the presence of flat parts of the boundary of $T$ that is not present when dealing with $T(1)$. Except that the structure of the optimum solutions is similar for both problems as we will see later. 
Clearly, $v c_{C}(G)=\frac{1}{C_{\mathrm{AND}}(G)}$, and a minimizer $x: V \rightarrow[0, \infty)$ realizing $v c_{C}(G)$ corresponds to a scaled capacity distribution $P$ that realizes $C_{\mathrm{AND}}(G)$. In what follows we will deal with the functional $v c_{C}(\cdot)$ rather than with $C_{\mathrm{AND}}(\cdot)$, as $v c_{C}(\cdot)$ appears to be additive with respect to certain natural decompositions of $G$.

\section{Strong crown decomposition}

In this section we recall some notions and results from our paper [2] trying to keep notation the same. Consider a graph $G=(V, E)$. For $U \subseteq V$, let $N(U)$ denote the set of its neighbors in $G, N(U):=\{v \in V: \exists u \in U$ such that $\{u, v\} \in E\}$, and $G[U]$ be the subgraph of $G$ induced by $U$.

Recall that a strong crown in a graph $G=(V, E)$ is a nonempty independent set $I$ of $G$ such that $|N(U) \cap I|>|U|$ holds for every nonempty set $U \subseteq N(I)$. If $I$ is a strong crown in $G$ then $I$ is the only maximum independent set in $G[I \cup N(I)]$ or even in the bipartite graph $G[I, N(I)]$ obtained from $G[I \cup N(I)]$ removing all edges within $N(I)$ (if any). It turns out that graphs containing a strong crown can be recognized efficiently and its strong crown can be found efficiently. As it may not be unique our aim is to find a maximal one.

For any graph $G=(V, E)$ there is a unique strong crown decomposition $(I, H, K)$, where $I$ is a strong crown, $H=N(I)$ and $K=V \backslash(I \cup H)$ is such that $G[K]$ contains no strong crowns. We will refer to $(I, H, K)$ as the canonical strong crown decomposition of $G$ in what follows (see [2] for more details).

We have $I=\emptyset$ in the decomposition above exactly when $(I, H, K)=(\emptyset, \emptyset, V)$, which is equivalent to the graph $G=(V, E)$ being Hallian; this means it satisfies Hall's property $|N(I)| \geq|I|$ for each independent set $I$ of $G$ or, equivalently, $|N(U)| \geq|U|$ for each $U \subseteq V$. In particular, the graphs $G[K]$ obtained by this decomposition always satisfy the Hall's property.

In questions of conjunctive capacity studied in this paper, Hallian graphs appear to be trivial. Nontrivial graphs are those with a nontrivial strong crown part $G[I \cup H]$ in their canonical strong crown decomposition.

\section{Binding number and binding set of a graph}

The concept of the binding number of a graph was introduced by Woodall [12] in 1973. The binding number of a graph $G=(V, E)$, denoted bind $(G)$, is given by

$$
\operatorname{bind}(G)=\min _{U \subseteq V}\left\{\frac{|N(U)|}{|U|}: U \neq \emptyset, N(U) \neq V\right\} .
$$

A binding set of $G$ is any set $U$ in $G$ with $\operatorname{bind}(G)=\frac{|N(U)|}{|U|}$.

There has been an increased interest in binding numbers as they may be related to other important graph properties. For example, if $\operatorname{bind}(G) \geq \frac{3}{2}$ and $G$ has at least three vertices then $G$ has a Hamiltonian circuit.

The binding number and the binding set can be computed in polynomial time ([4]). The approach to computing $\operatorname{bind}(G)$ is based on a standard idea 
for ratio minimization. Let's consider the problem of minimizing the difference $|N(U)|-\lambda|U|$, for $\lambda \geq 0$ a fixed number. If $d(G, \lambda)$ denotes $\min \{|N(U)|-\lambda|U|$ : $U \subseteq V, U \neq \emptyset, N(U) \neq V\}$, it is easy to see that $\operatorname{bind}(G) \geq \lambda$ if and only if $d(G, \lambda) \geq 0$.

Since $\operatorname{bind}(G)$ is a rational number whose numerator and denominator are bounded by $|V|$, we can deal with $\lambda$ of this form only. The corresponding minimization problem can be solved by a network flow method, but the condition $N(U) \neq V$ makes the problem difficult. We point out that in the situation of our main interest, namely, $\operatorname{bind}(G)<1, \operatorname{bind}(G)$ can be computed faster with one minimum cut calculation in time $O(\log |V|)$. The reason behind this is that if $\lambda<1$, the restriction that $N(U) \neq V$ can be dropped from the definition of $d(G, \lambda)$ without changing its value (if there is a binding set $U$ with property $N(U)=V$ then $\operatorname{bind}(G) \geq 1)$.

It is more relevant to focus our attention to the problem of determining the truncated version of the binding number (and binding set) problem, namely $\min \{\operatorname{bind}(G), 1\}$. Given a graph $G$, we either conclude that $\operatorname{bind}(G) \geq 1$ or, if $\operatorname{bind}(G)<1$, we want to find inclusionwise maximal binding set $U$. If $\operatorname{bind}(G)<$ 1 then every binding set $U$ of $G$ is an independent set of $G([8])$. In the following lemma we prove some important properties of binding sets.

Lemma 1. Let $G$ be a graph and let $(I, H, K)$ be its canonical strong crown decomposition. If bind $(G)<1$ then

(i) every binding set of $G$ is contained in $I$,

(ii) whenever $U$ and $W$ are two binding sets of $G$ then $U \cup W$ is a binding set, and $U \cap W$ is either empty or a binding set; in particular, the union of all binding sets of $G$ is a binding set.

The proof can be found in the Appendix.

Remark 1. Given any graph $G=(V, E)$ with $\operatorname{bind}(G)<1$, it is an important partial problem to find the unique inclusionwise maximal binding set $I^{*}$ of $G$. We know that we can compute efficiently a binding set $I_{1}$, let $H_{1}=N\left(I_{1}\right)$ and $G_{1}=G\left[V \backslash\left(I_{1} \cup H_{1}\right)\right]$. One can observe that $\operatorname{bind}\left(G_{1}\right) \geq \operatorname{bind}(G)$ (otherwise for

a binding set $I_{2}$ of $G_{1}$ setting $H_{2}=N_{G_{1}}\left(I_{2}\right)$ we would get $\frac{\left|H_{1} \cup H_{2}\right|}{\left|I_{1} \cup I_{2}\right|}<\operatorname{bind}(G)$, a contradiction).

Moreover, $\operatorname{bind}\left(G_{1}\right)>\operatorname{bind}(G)$ holds if and only if $I_{1}=I^{*}$. Firstly, if $\operatorname{bind}\left(G_{1}\right)=\operatorname{bind}(G)$ we get that $\frac{\left|H_{1} \cup H_{2}\right|}{\left|I_{1} \cup I_{2}\right|}=\operatorname{bind}(G)$, so $I_{1} \cup I_{2}$ is a larger binding set of $G$ and, in particular, $I_{1}$ cannot be whole of $I^{*}$. Secondly, if $I_{1} \neq I^{*}$ then $\operatorname{bind}\left(G_{1}\right)=\operatorname{bind}(G)$. To show that, it is sufficient to show $\operatorname{bind}\left(G_{1}\right) \leq \operatorname{bind}(G)$. Setting $H^{*}=N\left(I^{*}\right), b:=\operatorname{bind}(G)$, we have $\left|H^{*}\right|=b\left|I^{*}\right|,\left|H_{1}\right|=b\left|I_{1}\right|$ and consequently $N_{G_{1}}\left(I^{*} \backslash I_{1}\right)=H^{*} \backslash H_{1}$ and $\left|H^{*} \backslash H_{1}\right|=b\left|I^{*} \backslash I_{1}\right|$, showing $\operatorname{bind}\left(G_{1}\right) \leq \operatorname{bind}(G)$.

Now we can set $G_{2}=G\left[V \backslash\left(I_{1} \cup I_{2} \cup H_{1} \cup H_{2}\right)\right]$. If $I_{1} \cup I_{2}$ is not $I^{*}$, i.e. $\operatorname{bind}\left(G_{2}\right)=\operatorname{bind}(G)$, for a binding set $I_{3}$ of $G_{2}$ that we then compute with $H_{3}=N_{G_{2}}\left(I_{3}\right)$ we get that $I_{1} \cup I_{2} \cup I_{3}$ is a larger binding set of $G$. We can continue in this way until $I_{1} \cup I_{2} \cup \cdots \cup I_{k}$ will be that maximal binding set $I^{*}$ of $G$. 


\section{Minimum Capacitary Vertex Cover and its properties}

Now we study the properties of the optimal solutions of the Minimum Capacitary Vertex Cover problem. Recall that this is a convex programming problem defined on a graph $G=(V, E)$ by

$$
v c_{C}(G)=\min _{x}\{x(V):(x(u), x(v)) \in T(1) \text { for every }\{u, v\} \in E\} .
$$

Here $T(1)=\{(a, b) \in[0, \infty) \times[0, \infty): f(a, b) \geq 1\}$, where $f(x, y)=(x+$ $y) \log (x+y)-x \log x-y \log y$ for $x, y>0$, extended continuously to $[0, \infty) \times$ $[0, \infty)$.

It is important that $f$ is concave on $(0, \infty) \times(0, \infty)$. To verify this, we can compute the Hessian matrix $H(x, y)$ of $f(x, y)$ which reads as

$H(x, y)=\left(\begin{array}{cc}\frac{1}{x+y}-\frac{1}{x}, & \frac{1}{x+y} \\ \frac{1}{x+y}, & \frac{1}{x+y}-\frac{1}{y}\end{array}\right)$ and so $\operatorname{det}(\lambda I-H(x, y))=\lambda\left(\lambda+\frac{1}{x}+\frac{1}{y}-\frac{2}{x+y}\right)$.

As its eigenvalues are non-positive, $H(x, y)$ is negative semidefinite, and so $f(x, y)$ is concave. Consequently, $T(1)$ is a convex set.

Let us denote by $\varphi(x)$ the function whose graph describes the boundary $\partial T(1)$. For any $x \in(0, \infty), \varphi(x)$ denotes a unique $y=\varphi(x)$ such that $(x+$ y) $\log (x+y)-x \log x-y \log y=1$. As $T(1)$ is symmetric with respect to the line $y=x, \varphi(\varphi(x))=x$ for each $x \in(0, \infty)$. Moreover, $\varphi\left(\frac{1}{2}\right)=\frac{1}{2}$, and $\varphi:(0, \infty) \rightarrow$ $(0, \infty)$ is a real analytic function. We can observe that $\partial T(1)$ does not contain any line segment, as the uniqueness theorem for real analytic functions would allow such behaviour of $\varphi$ only for functions that are affine globally in $(0, \infty)$, which $\varphi$ certainly is not. We can thus conclude that $T(1)$ is strictly convex. The fact that its boundary does not contain any line segments will be important in our proof that the optimal solution to the Minimum Capacitary Vertex Cover problem is unique.

Note. It is sometimes useful to have $\varphi(x)$ parametrized by the ratio $t=\frac{\varphi(x)}{x}$. Given any $t \in(0, \infty)$, as $f(x, t x)$ simplifies to $x((1+t) \log (1+t)-t \log t), \stackrel{x}{1}=$ $f(x, x t)$ implies $x=\frac{1}{(1+t) \log (1+t)-t \log t}$. It is easy to check that with decreasing $t \in(0, \infty)$ this $x \in(0, \infty)$ increases. So $\partial T(1)$ parametrized by $t=\frac{\varphi(x)}{x}$ reads as

$$
\partial T(1)=\left\{\left(\frac{1}{(1+t) \log (1+t)-t \log t}, \frac{t}{(1+t) \log (1+t)-t \log t}\right): t \in(0, \infty)\right\} .
$$

Moreover, differentiating $(x+\varphi(x)) \log (x+\varphi(x))-x \log x-\varphi(x) \log \varphi(x)=1$ we can derive that

$$
\varphi^{\prime}(x)=-\frac{\log \left(1+\frac{\varphi(x)}{x}\right)}{\log \left(1+\frac{x}{\varphi(x)}\right)},
$$

from which it is pretty obvious that $\varphi^{\prime}:(0, \infty) \rightarrow(-\infty, 0)$ smoothly increases from $-\infty$ to 0 for $x$ varying from 0 to $+\infty$. 
Let $G=(V, E)$ be a graph. It is clear that a minimum capacitary vertex cover $x: V \rightarrow[0, \infty)$ for $G$ achieves value 0 at each isolated vertex of $G$. Removing all isolated vertices of $G$ (if any) will reduce the problem to the equivalent one on a graph without isolated vertices. The following lemma is a starting point to the study of exact solutions to the Minimum capacitary vertex cover problem.

Lemma 2. Let $G=(V, E)$ be a graph without isolated vertices and $x: V \rightarrow$ $(0, \infty)$ be a minimum capacitary vertex cover for $G$. Then the following hold:

(i) $E^{x}:=\{\{u, v\} \in E: f(x(u), x(v))=1\}$ is an edge cover of $G$.

(ii) Let $V_{<}^{x}:=\left\{u \in V: x(u)<\frac{1}{2}\right\}, V_{>}^{x}:=\left\{u \in V: x(u)>\frac{1}{2}\right\}$, and

$V_{=}^{x}:=\left\{u \in V: x(u)=\frac{1}{2}\right\}$. Then $V_{<}^{x}$ is an independent set in $G$ and $N\left(V_{<}^{x}\right)=V_{>}^{x}$.

(iii) Every connected component $F=(V(F), E(F))$ of the graph $\left(V, E^{x}\right)$ is either the component of $G\left[V_{=}^{x}\right]$, or the component of the bipartite graph $G\left[V_{<}^{x}, V_{>}^{x}\right]$. In the latter case there exists $q \in\left(0, \frac{1}{2}\right)$ (depending on $F$ ) such that

$$
x(u)= \begin{cases}q, & \text { if } u \in V(F) \cap V_{<}^{x} \\ \varphi(q), & \text { if } u \in V(F) \cap V_{>}^{x} .\end{cases}
$$

The proof can be found in the Appendix.

Theorem 1. For any graph $G=(V, E)$ the minimum capacitary vertex cover for $G$ is unique.

The proof can be found in the Appendix.

\section{Auxiliary Two-valued Capacitary Vertex Cover}

While for any graph $G=(V, E)$ the function $x \equiv \frac{1}{2}$ on $V$ is always a feasible capacitary vertex cover, there are situations when this choice of $x$ would clearly be suboptimal; for example, if $G$ contains isolated vertices.

In view of part (iii) of Lemma 2 , it is interesting to consider (minimum) capacitary vertex covers that assume exactly two different values on the vertex set of a graph without isolated vertices. More specifically, let $q<\frac{1}{2}$ and $p=$ $\varphi(q)>\frac{1}{2}$ be these two values of a minimum capacitary vertex cover $x$, and let $I \cup H$ be the partition of $V$ such that $\left.x\right|_{I}=q$ and $\left.x\right|_{H}=p$. Then, clearly, $I$ is an independent set in $G$ and we show that $|I|>\frac{|V|}{2}$.

More generally, for any partition $I \cup H$ of $V$ into two nonempty sets we can consider the following auxiliary two-valued minimization problem $s:=\min \{q|I|+$ $p|H|:(q, p) \in T(1)\}$. Let us consider the lines $L_{R}=\{(q, p): q|I|+p|H|=R\}$ for any real $R$. They all have a slope $-\frac{|I|}{|H|}$ and exactly one of them touches the boundary of $T(1)$. The point of this touching, $(x, \varphi(x))$ has to satisfy $\varphi^{\prime}(x)=-\frac{|I|}{|H|}$.

As we observed earlier, $\varphi^{\prime}(x)=-\frac{\log \left(1+\frac{\varphi(x)}{x}\right)}{\log \left(1+\frac{x}{\varphi(x)}\right)}$, so in terms of the parameter $t=\frac{\varphi(x)}{x}$, this point of touching corresponds to the unique root $t$ (denoted as 
$t=F\left(\frac{|H|}{|I|}\right)$, as it depends on the ratio $\frac{|H|}{|I|}$ only) to the equation $\frac{\log (1+t)}{\log \left(1+\frac{1}{t}\right)}=\frac{|I|}{|H|}$ (or, equivalently, $\left.1-\frac{|H|}{|I|}=\frac{\log t}{\log (t+1)}\right)$.

The optimal solution $s$ to the above minimization problem will then read in terms of $F\left(\frac{|H|}{|I|}\right)$ as

$$
s=\frac{|I|}{\log \left(1+F\left(\frac{|H|}{|I|}\right)\right)}=\frac{|H|}{\log \left(1+\frac{1}{F\left(\frac{|H|}{|I|}\right)}\right)} .
$$

In another words, this minimum is the unique real root $s$ to the equation $2^{-\frac{|H|}{s}}+$ $2^{-\frac{|I|}{s}}=1$. In addition to $F\left(\frac{|H|}{|I|}\right)$, we will denote by $\Psi\left(\frac{|H|}{|I|}\right)$ that $(q, p)$ that minimizes $s$ above, hence the point of touch. Namely, $\Psi\left(\frac{|H|}{|I|}\right)$ is the point

$$
\left(\frac{1}{\left(1+F\left(\frac{|H|}{|I|}\right)\right) \log \left(1+F\left(\frac{|H|}{|I|}\right)\right)-F\left(\frac{|H|}{|I|}\right) \log F\left(\frac{|H|}{|I|}\right)}, \frac{F\left(\frac{|H|}{|I|}\right)}{\left(1+F\left(\frac{|H|}{|I|}\right)\right) \log \left(1+F\left(\frac{|H|}{|I|}\right)\right)-F\left(\frac{|H|}{|I|}\right) \log F\left(\frac{|H|}{|I|}\right)}\right)
$$

the unique point on $\partial T(1)$ with the slope $-\frac{|I|}{|H|}$.

This auxiliary problem makes perfect sense for any partition $I \cup H$ of $V$ into two nonempty sets, but it is related to the minimum capacitary vertex cover problem only under some additional assumptions. By symmetry, we could confine ourselves to the case when $|I| \geq|H|$, so that point $(q, p)=\Psi\left(\frac{|H|}{|I|}\right)$ of $\partial T(1)$ with the slope $-\frac{|I|}{|H|}$ will have $q \leq \frac{1}{2}$. As $|I|=|H|$ corresponds to the touching point $(p, q)=\left(\frac{1}{2}, \frac{1}{2}\right)$, to achieve a two-valued solution we assume $|I|>|H|$, leading to $q<\frac{1}{2}$.

If one defines $\left.x\right|_{I}=q$ and $\left.x\right|_{H}=p$ then it will be a feasible capacitary vertex cover only if $I$ is an independent set in $G$, otherwise the constraints $(x(u), x(v)) \in T(1)$ for any edge with both vertices $u$ and $v$ in $I$, are not met.

We can then observe that a necessary condition for a graph $G=(V, E)$ to have a two-valued minimum capacitary vertex cover is to have an independent set $I$ of size $>\frac{|V|}{2}$. Later (in Theorem 4) we will be able to provide necessary and sufficient conditions for $G$ to have a two valued minimum capacitary vertex cover.

\section{Minimum Fractional and Capacitary Vertex Covers}

It is easy to see that any capacitary vertex cover for $G=(V, E)$ is a fractional vertex cover for $G$ (as $T(1) \subseteq T$, see Fig. 1 ), so $v c_{*}(G) \leq v c_{C}(G)$. Moreover, the uniform function $x \equiv \frac{1}{2}$ on $V$ is always a feasible capacitary vertex cover. Consequently, $v c_{*}(G) \leq v c_{C}(G) \leq \frac{|V|}{2}$. In terms of the conjunctive capacity,

$$
\frac{2}{|V|} \leq C_{\mathrm{AND}}(G) \leq \frac{1}{v c_{*}(G)}
$$

The Hallian graphs, i.e. those graphs that contain a system of vertex disjoint edges and (odd) cycles covering all the vertices are extremals for these bounds. 
Namely, assuming that a graph $G=(V, E)$ does not have isolated vertices, $v c_{*}(G)=\frac{|V|}{2}$ if and only if $G$ is Hallian.

In the following theorem we prove that a similar result also holds for the Minimum Capacitary Vertex problem (the proof can be found in the Appendix).

Theorem 2. Let $G=(V, E)$ be a graph without isolated vertices, then $v_{C}(G)=$ $\frac{|V|}{2}$ if and only if $G$ is Hallian.

\section{The structure of Minimum Capacitary Vertex Covers}

Now we describe in detail how the unique minimum capacitary vertex cover $x: V \rightarrow(0, \infty)$ for a graph $G=(V, E)$ without isolated vertices can look like. Let $(I, H, K)$ be the canonical strong crown decomposition of $G$. We show that then $V_{<}^{x}=I, V_{>}^{x}=H$, and $V_{=}^{x}=K$. Moreover, we describe how the exact values of $x$ on $I \cup H$ can be found by computing binding sets of certain graphs.

Theorem 3. Let $G=(V, E)$ be a graph without isolated vertices with the canonical strong crown decomposition $(I, H, K)$ and let $x: V \rightarrow(0, \infty)$ be the minimum capacitary vertex cover for $G$. Assume that $\left.x\right|_{H}$ achieves $m \geq 1$ values, $p_{1}>p_{2}>\cdots>p_{m}$. Then $p_{m}>\frac{1}{2}$, and $\left.x\right|_{I}$ achieves $m$ values $q_{1}=\varphi\left(p_{1}\right)<$ $q_{2}=\varphi\left(p_{2}\right)<\cdots<q_{m}=\varphi\left(p_{m}\right)<\frac{1}{2}$.

Put $I_{i}:=\left\{v \in I: x(v)=q_{i}\right\}, H_{i}:=\left\{v \in H: x(v)=p_{i}\right\}$ for $i=$ $1,2, \ldots, m$. Then $H_{i} \subseteq N\left(I_{i}\right) \subseteq \cup_{j=1}^{i} H_{j}$ and $I_{i} \subseteq N\left(H_{i}\right) \cap I \subseteq \cup_{j=i}^{m} I_{j}$ for each $i=1,2, \ldots, m$.

The ratio $\frac{\left|H_{i}\right|}{\left|I_{i}\right|}$ is uniquely determined by the values $\left(q_{i}, p_{i}\right)$, namely $\left(q_{i}, p_{i}\right)=$ $\Psi\left(\frac{\left|H_{i}\right|}{\left|I_{i}\right|}\right)$. Moreover, for each connected component $F=(V(F), E(F))$ of the graph $\left(V, E^{x}\right)$ with $V(F) \subseteq I_{i} \cup H_{i}$ we have the same ratio $\frac{\left|V(F) \cap H_{i}\right|}{\left|V(F) \cap I_{i}\right|}=\frac{\left|H_{i}\right|}{\left|I_{i}\right|}$, as $\left(q_{i}, p_{i}\right)=$ $\Psi\left(\frac{\left|V(F) \cap H_{i}\right|}{\left|V(F) \cap I_{i}\right|}\right)$ holds.

In particular, $\frac{\left|H_{1}\right|}{\left|I_{1}\right|}<\frac{\left|H_{2}\right|}{\left|I_{2}\right|}<\cdots<\frac{\left|H_{m}\right|}{\left|I_{m}\right|}<1$.

The proof can be found in the Appendix.

We are now ready to describe graphs $G=(V, E)$ for which a two-valued capacitary vertex cover is optimal. Such graphs have their canonical strong crown decomposition $(I, H, K)$ with the Hallian part $K$ empty and with $I$ a binding set of $G$. In other words one could describe such graphs $G=(V, E)$ as those with $\alpha(G)>\frac{|V|}{2}$ and such that for each non-empty independent set $U$ in $G$ $\frac{|N(U)|}{|U|} \geq \frac{\tau(G)}{\alpha(G)}$. Here $\alpha(G)$ is the size of the maximum independent set and $\tau(G)$ is the size of the minimum vertex cover in the graph $G$.

Theorem 4. Let $G=(V, E)$ be a graph without isolated vertices and with the canonical strong crown decomposition $(I, H, \emptyset)$ and assume that $I \neq \emptyset$ is a binding set of $G$. Then the minimum capacitary vertex cover $x: V \rightarrow(0, \infty)$ is two-valued, $\left.x\right|_{I}=q,\left.x\right|_{H}=p$, with $(q, p)=\Psi\left(\frac{|H|}{|I|}\right)$. 
The proof can be found in the Appendix.

Let $G=(V, E)$ be a graph without isolated vertices, let $(I, H, K)$ be its canonical strong crown decomposition and assume that $I \neq \emptyset($ so $\operatorname{bind}(G)<1)$. Let $I_{1}$ be the inclusionwise maximal binding set of $G$. As we observed earlier $I_{1} \subseteq I$. Put $H_{1}:=N\left(I_{1}\right)$ and $\left(q_{1}, p_{1}\right)=\Psi\left(\frac{\left|H_{1}\right|}{\left|I_{1}\right|}\right)$. Let us take $\left.x\right|_{I_{1}}=q_{1},\left.x\right|_{H_{1}}=p_{1}$. In the induced graph $G\left[I_{1} \cup H_{1}\right]$ we can conclude by the previous theorem that the minimum capacitary vertex cover is two-valued and achieves the values we have assigned them.

Now we study the question on the graph obtained by removing $I_{1}$ and $H_{1}$ from $G$. Denote $G_{1}=G\left[V \backslash\left(I_{1} \cup H_{1}\right)\right]$. It can be easily verified that $G_{1}$ has the canonical strong crown decomposition $\left(I \backslash I_{1}, H \backslash H_{1}, K\right)$. (It is sufficient to check that it is still $|N(U) \cap I|>|U|$ whenever $U$ is a nonempty subset of $H \backslash H_{1}$.)

If $I \backslash I_{1} \neq 0$ (so $\operatorname{bind}\left(G_{1}\right)<1$ ) we can take $I_{2}$, the inclusionwise maximal binding set of $G_{1}, H_{2}:=N_{G_{1}}\left(I_{2}\right)$ and put $\left(q_{2}, p_{2}\right)=\Psi\left(\frac{\left|H_{2}\right|}{\left|I_{2}\right|}\right)$. We take $\left.x\right|_{I_{2}}=q_{2}$, $\left.x\right|_{H_{2}}=p_{2}$. With this choice of $x$ in the induced graph $G\left[I_{2} \cup H_{2}\right], x$ defines the minimum capacitary vertex cover.

Observe now that $\operatorname{bind}(G)=\frac{\left|H_{1}\right|}{\left|I_{1}\right|}<\frac{\left|H_{2}\right|}{\left|I_{2}\right|}=\operatorname{bind}\left(G_{1}\right)$. If not, then $\frac{\left|H_{1} \cup H_{2}\right|}{\left|I_{1} \cup I_{2}\right|} \leq$ $\operatorname{bind}(G)$ and $I_{1} \cup I_{2}$ would be an inclusionwise larger binding set of $G$ than $I_{1}$, a contradiction. So

$$
\frac{\left|H_{1}\right|}{\left|I_{1}\right|}<\frac{\left|H_{2}\right|}{\left|I_{2}\right|}, \text { and as }\left(q_{i}, p_{i}\right)=\Psi\left(\frac{\left|H_{i}\right|}{\left|I_{i}\right|}\right), i=1,2, \ldots
$$

we conclude that $q_{1}<q_{2}<\frac{1}{2}$ and $p_{1}>p_{2}>\frac{1}{2}$.

We can continue in the same way and stop after $m$ steps once $\cup_{i=1}^{m} I_{i}$ exhausts $I$. The rest is the Hallian graph $G[K]$. For $K$ we take $\left.x\right|_{K} \equiv \frac{1}{2}$ as we know that for Hallian graphs it is the optimal value of the minimum capacitary vertex cover (Theorem 2). We have

$$
\frac{\left|H_{1}\right|}{\left|I_{1}\right|}<\frac{\left|H_{2}\right|}{\left|I_{2}\right|}<\cdots<\frac{\left|H_{m}\right|}{\left|I_{m}\right|}<1,\left(q_{i}, p_{i}\right)=\Psi\left(\frac{\left|H_{i}\right|}{\left|I_{i}\right|}\right),\left.x\right|_{I_{i}}=q_{i},\left.x\right|_{H_{i}}=p_{i},
$$

for $i=1,2, \ldots, m$ and $q_{1}<q_{2}<\cdots<q_{m}<\frac{1}{2}$.

If we define $x: V \rightarrow(0, \infty)$ this way, we have a potential solution that is locally optimal on each of $G\left[I_{1} \cup H_{1}\right], G\left[I_{2} \cup H_{2}\right], \ldots, G\left[I_{m} \cup H_{m}\right]$, and $G[K]$. So $v c_{C}(G)$ is at least $x(V)$ and to show that it is indeed $x(V)$ we need to check that $x$ is a feasible capacitary vertex cover for $G$ satisfying all the constraints $(x(u), x(v)) \in T(1)$ also for pairs $\{u, v\} \in E$ belonging to distinct pieces of $G\left[I_{1} \cup H_{1}\right], G\left[I_{2} \cup H_{2}\right], \ldots, G\left[I_{m} \cup H_{m}\right]$, and $G[K]$. For this it is sufficient to check that all vertices of $I$ that have assigned $x(u)<\frac{1}{2}$, have their neighbours $v$ with $x(v)$ large enough. If $u \in I_{i}$, say, then $x(u)=q_{i}$, and any neighbour $v$ of $u$ needs to have $x(v) \geq \varphi\left(q_{i}\right)=p_{i}$ to comply with the constraints, so only $v \in \cup_{j=1}^{i} H_{j}$ would be allowed. But our construction ensures that $H_{i} \subseteq N\left(I_{i}\right) \subseteq \cup_{j=1}^{i} H_{j}$ for each $i=1,2, \ldots, m$, so there are no edges from $u \in I_{i}$ to any $v \in H_{j}$ with $j>i$. Hence our $x$ is indeed a feasible capacitary vertex cover for $G$ and consequently it is the minimum capacitary vertex cover for $G$. 
We can summarise the facts that we have just explained in the following theorem.

Theorem 5. Let $G=(V, E)$ be a graph without isolated vertices and with the canonical strong crown decomposition $(I, H, K)$, and let $x: V \rightarrow(0, \infty)$ be the (unique) minimum capacitary vertex cover for $G$. Then $I=\left\{v \in V: x(v)<\frac{1}{2}\right\}$, $H=\left\{v \in V: x(v)>\frac{1}{2}\right\}$ and $K=\left\{v \in V: x(v)=\frac{1}{2}\right\}$. If $G$ is not Hallian, then $\left.x\right|_{I}$ has $m \geq 1$ values $q_{1}<q_{2}<\ldots q_{m}<\frac{1}{2}$ and $\left.x\right|_{H}$ has $m$ values $p_{1}=$ $\varphi\left(q_{1}\right)>p_{2}=\varphi\left(q_{2}\right)>\cdots>p_{m}=\varphi\left(q_{m}\right)>\frac{1}{2}$ with $I_{i}:=\left\{u \in I: x(v)=q_{i}\right\}$, $H_{i}:=\left\{v \in H: x(v)=p_{i}\right\}$. Here $I_{1}$ is the inclusionwise maximal binding set for $G, H_{1}=N\left(I_{1}\right)$ and $\left(q_{1}, p_{1}\right)=\Psi\left(\frac{\left|H_{1}\right|}{\left|I_{1}\right|}\right)$. Moreover, after removing the vertices $I_{1} \cup H_{1}$ from the graph, $\left.x\right|_{V \backslash\left(I_{1} \cup H_{1}\right)}$ is still a minimum capacitary vertex cover in the rest $G\left[V \backslash\left(I_{1} \cup H_{1}\right)\right]$. We can find all pairs $\left(I_{i}, H_{i}\right)$ of sets and their corresponding values $\left(q_{i}, p_{i}\right), i=1,2, \ldots, m$, by repeatedly computing the inclusionwise maximal binding set $I_{i}$ in $G_{i-1}:=G\left[V \backslash \cup_{j<i}\left(I_{j} \cup H_{j}\right)\right], H_{i}=$ $N_{G_{i-1}}\left(I_{i}\right)$, and $\left(q_{i}, p_{i}\right)=\Psi\left(\frac{\left|H_{i}\right|}{\left|I_{i}\right|}\right)$.

Remark 2. As the convex programming problem of finding the minimum capacitary vertex cover problem is efficiently computable one can use it as an alternative way of computing the canonical strong crown decomposition $(I, H, K)$ of any graph $G=(V, E)$ without isolated vertices, given that $I=V_{<}^{x}, H=V_{>}^{x}$, and $K=V_{=}^{x}$.

\section{References}

1. C. Berge, Graphs, North-Holland, Amsterdam, 1985.

2. M. Chlebík, J. Chlebíková, Crown reductions for the Minimum Weighted Vertex Cover problem, Discrete Applied Mathematics 156(2008), 292-312.

3. G. Cohen, J. Körner, and G, Simonyi, Zero-error capacities and very different sequences, in Sequences: combinatorics, compression, security and transmission, R. M. Capocelli (Ed.), Springer-Verlag, 1990, pp. 144-155.

4. W. H. Cunningham, Computing the binding number of a graph, Discr. Appl. Math 27(1990), 283-285.

5. L. Gargano, J. Körner, and U. Vaccaro, Sperner capacities, Graphs and Combinatorics 9(1993), 31-46.

6. L. Gargano, J. Körner, and U. Vaccaro, Capacities: from information theory to extremal set theory, J. Comb. Theory Ser. A 68(1994), 296-316.

7. G. Greco, Capacities of graphs and 2-matchings, Discrete Mathematics 186(1998), 135-143.

8. V. G. Kane, S. P. Mohanty, E. G. Straus, Which rational numbers are binding numbers?, Journal of Graph Theory 5(1981), 379-384.

9. J. Körner, G. Simonyi, A Sperner-type theorem and qualitative independence, J. Comb. Theory 59(1992), 90-103.

10. A. Rényi, Probability theory, North-Holland, Amsterdam/New York, 1970.

11. C. E. Shannon, The zero-error capacity of a noisy channel, IRE Trans. Inform. Theory 2,(1956), 8-19.

12. D. R. Woodal, The binding number of a graph and its Anderson number, J. Combin. Theory Ser. B 15(1973), 225-255. 


\section{Appendix}

\section{The proof of Lemma 1}

Proof. (i) Let $U$ be a binding set of $G$. If $\operatorname{bind}(G)<1$ then $U$ is an independent set, as observed in [8]. Put $U_{I}=U \cap I, U_{H}=U \cap H$ and $U_{K}=U \cap K$. Let us prove that $U_{H}=\emptyset$. Assume, on contrary, that $U_{H} \neq \emptyset$. As then $\left|N\left(U_{H}\right) \cap I\right|>\left|U_{H}\right|$ we have $U \backslash U_{H} \neq \emptyset$ and

$$
\begin{gathered}
\left|N\left(U \backslash U_{H}\right)\right| \leq|N(U)|-\left|N\left(U_{H}\right) \cap I\right|<|N(U)|-\left|U_{H}\right|, \quad \text { so } \\
\frac{\left|N\left(U \backslash U_{H}\right)\right|}{\left|U \backslash U_{H}\right|}<\frac{|N(U)|-\left|U_{H}\right|}{|U|-\left|U_{H}\right|} \leq \frac{|N(U)|}{|U|},
\end{gathered}
$$

a contradiction. So $U_{H}=\emptyset$.

Let us now prove that $U_{K}=\emptyset$. Assume, on contrary, that $U_{K} \neq \emptyset$. Then

$$
\begin{aligned}
& \left|N\left(U_{I}\right)\right| \leq|N(U)|-\left|N\left(U_{K}\right) \cap K\right| \leq|N(U)|-\left|U_{K}\right| \text { so } \\
& \frac{\left|N\left(U_{I}\right)\right|}{\left|U_{I}\right|}=\frac{\left|N\left(U \backslash U_{K}\right)\right|}{\left|U \backslash U_{K}\right|} \leq \frac{|N(U)|-\left|U_{K}\right|}{|U|-\left|U_{K}\right|}<\frac{|N(U)|}{|U|}
\end{aligned}
$$

assuming $\operatorname{bind}(G)<1$ and $U_{K} \neq 0$, a contradiction. So $U_{K}=\emptyset$. Consequently, $U=U_{I} \subseteq I$.

(ii) Assume now that $U, W$ are two binding sets of $G$ (hence $U, W \subseteq I$ by (i)) and denote $b:=\operatorname{bind}(G)$. Then we have $|N(U)|=b|U|$ and $|N(W)|=b|W|$.

Obviously,

$$
\begin{aligned}
b(|U \cup W|+|U \cap W|) & =b(|U|+|W|)=|N(U)|+|N(W)| \\
& =|N(U) \cup N(W)|+|N(U) \cap N(W)| .
\end{aligned}
$$

But

$|N(U) \cup N(W)|=|N(U \cup W)| \geq b|U \cup W|$, and $|N(U) \cap N(W)| \geq|N(U \cap W)| \geq b|U \cap W|$, so the formula (6) can continue with $\geq b(|U \cup W|+|U \cap W|)$, and so equality holds everywhere above.

In particular,

$$
|N(U) \cap N(W)|=|N(U \cap W)|=b|U \cap W| \text { and }|N(U \cup W)|=b|U \cup W| .
$$

So $U \cap W$ is either empty or a binding set, and $U \cup W$ is a binding set.

\section{The proof of Lemma 2}

Proof. (i) Assume that $E^{x}$ is not an edge cover of $G$. Then there exists $u \in V$ that is not covered by $E^{x}$ and for this $u \in V$ we have $f(x(u), x(v))>1$ for all 
$v \in V$ such that $\{u, v\} \in E$. Clearly, by continuity, there exists $\delta>0$ such that $(x(u)-\delta, x(v)) \in T(1)$ for all $v \in V$ such that $\{u, v\} \in E$. But then

$$
x_{\delta}(w)= \begin{cases}x(w)-\delta, & w=u \\ x(w), & w \in V \backslash\{u\}\end{cases}
$$

is a feasible capacitary vertex cover with less total weight $x_{\delta}(V)=x(V)-$ $\delta$, a contradiction. Our assumption that $E^{x}$ is not an edge cover leads to a contradiction, so $E^{x}$ has to be an edge cover of $G$.

(ii) If $u \in V_{<}^{x}$ then $x(u)<\frac{1}{2}$, and $(x(u), y)$ belongs to a feasible set $T(1)$ for $y \geq \varphi(x(u))>\frac{1}{2}$ only. So $N\left(V_{<}^{x}\right) \subseteq V_{>}^{x}$ and $V_{<}^{x}$ is an independent set of $G$. Moreover, as each $v \in V_{>}^{x}$ has to be covered by some edge $E^{x}, v \in N\left(V_{<}^{x}\right)$ and $N\left(V_{<}^{x}\right)=V_{>}^{x}$ follows.

(iii) Consider a connected component $F=(V(F), E(F))$ of the graph $\left(V, E^{x}\right)$ which is not a component of the graph $G\left[V_{=}^{x}\right]$. Let $u \in V(F)$ be such that $x(u)=p \neq \frac{1}{2}$. As $u$ has to be covered by $E^{x}$, there is its neighbour $v \in V(F)$ with $x(v)=\varphi(p)$. In the graph $\left(G, E^{x}\right)$, a vertex assigned $p$ can only be connected to vertices assigned $\varphi(p)$; and a vertex assigned $\varphi(p)$ can only be connected to vertices assigned $p$. It easily follows that $x$ assumes only two different values on the vertices of $F$, namely $p$ and $\varphi(p)$.

\section{The proof of Theorem 1}

Proof. We can assume without any loss of generality that $G$ does not contain isolated vertices. Assume, on contrary, that $x_{1}, x_{2}: V \rightarrow(0, \infty)$ are two distinct minimum capacitary vertex covers, and $u \in V$ is such that $x_{1}(u) \neq x_{2}(u)$. As the set $T(1)$ is convex, the choice $x:=\frac{1}{2}\left(x_{1}+x_{2}\right)$ is also a feasible capacitary vertex cover for $G$. As $x_{1}(V)=x_{2}(V)=v c_{C}(G)$, we also have $x(V)=v c_{C}(G)$ and so $x$ is a minimum capacitary vertex cover for $G$. By Lemma 2 part (i), the edges $E^{x}$ create an edge cover of $G$. But we will show that this is not the case here and so obtain a contradiction.

Consider any $v \in V$ such that $\{u, v\} \in E$. As $A_{1}:=\left(x_{1}(u), x_{1}(v)\right)$ and $A_{2}:=\left(x_{2}(u), x_{2}(v)\right)$ are two distinct points of a convex set $T(1)$, and as we observed earlier, the boundary $\partial T(1)$ of $T(1)$ does not contain any line segment, the middle point $(x(u), x(v))$ of the segment $A_{1} A_{2}$ belongs to the interior of $T(1)$, and not to its boundary. Consequently, $f(x(u), x(v))>1$ for every $v \in V$ such that $\{u, v\} \in E$, and so $u$ is not covered by the edge of $E^{x}$, a contradiction. Our assumption that there are two distinct minimum capacitary vertex covers leads to a contradiction, so this solution has to be unique.

\section{The proof of Theorem 2}

Proof. If $G$ is Hallian then $v c_{*}(G)=\frac{|V|}{2}$ is well known (see e.g. [2]), so our sandwitch estimates $v c_{*}(G) \leq v c_{C}(G) \leq \frac{|V|}{2}$ imply that $v c_{C}(G)=\frac{|V|}{2}$ then. Assume now that $G=(V, E)$ is a non-Hallian graph without isolated vertices. 
Then there exists an independent set $I$ in $G$ with $|N(I)|<|I|$. In this new graph $G[I \cup N(I)]$ induced by $I \cup N(I)$ a two-valued solution $(q, p)=\Psi\left(\frac{|N(I)|}{|I|}\right)$ described above defines easily a feasible solution showing $v c_{C}(G)<\frac{|V|}{2}$. Namely we can take $x: V \rightarrow(0, \infty)$

$$
x(v)= \begin{cases}q, & v \in I \\ p, & v \in N(I) \\ \frac{1}{2}, & v \in V \backslash(I \cup N(I)) .\end{cases}
$$

\section{The proof of Theorem 3}

Proof. (a) First let us assume that $K=\emptyset$ and that there are no edges within $H$, so $G$ is bipartite. Let $x: V \rightarrow(0, \infty)$ be the minimum capacitary vertex cover for $G$ and $\left.x\right|_{H}$ have $m \geq 1$ values, $p_{1}>p_{2}>\cdots>p_{m}$, with $H_{i}:=\{v \in H$ : $\left.x(v)=p_{i}\right\}, i=1,2, \ldots, m$, defining the partition of $H$. As edges $E^{x}$ cover all the vertices $V$ (by Lemma 2), $\left.x\right|_{I}$ has the values $q_{1}=\varphi\left(p_{1}\right)<q_{2}=\varphi\left(p_{2}\right)<$ $\cdots<q_{m}=\varphi\left(p_{m}\right)$ only, with $I_{i}=\left\{v \in I: x(v)=q_{i}\right\}$.

We can easily conclude using edge constraints of capacitary vertex covers that

$$
\begin{aligned}
H_{i} & \subseteq N\left(I_{i}\right) \subseteq \cup_{j=1}^{i} H_{j}, \\
I_{i} & \subseteq N\left(H_{i}\right) \cap I \subseteq \cup_{j=i}^{m} I_{j}, \quad \text { for all } i=1,2, \ldots, m .
\end{aligned}
$$

In particular, $I_{m}=N\left(H_{m}\right) \cap I$, and using properties of strong crown decompositions, $\left|I_{m}\right|>\left|H_{m}\right|$, so $\frac{\left|H_{m}\right|}{\left|I_{m}\right|}<1$ follows.

To prove that $p_{m}>\frac{1}{2}$ assume, on contrary, that $p_{m} \leq \frac{1}{2}$. But then obviously the line

$$
\left\{(q, p): q\left|I_{m}\right|+p\left|H_{m}\right|=q_{m}\left|I_{m}\right|+p_{m}\left|H_{m}\right|\right\}
$$

with slope $-\frac{\left|I_{m}\right|}{\left|H_{m}\right|}<-1$ is not tangent to $\partial T(1)$ (assuming $p_{m} \leq \frac{1}{2}$, in which range tangents have slope $\geq-1)$. Consequently, a small perturbation $(q, p)$ of $\left(q_{m}, p_{m}\right)$ can decrease the value of a capacitary vertex cover. If we put $\left.x\right|_{I_{m}}=$ $q=q_{m}-\varepsilon,\left.x\right|_{H_{m}}=\varphi(q)$ for some $q$ very close to $q_{m}$, all constraints of edges but $E^{x}$ will still read as $f(x(u), x(v))>1$, by a simple continuity argument. So, the assumption $p_{m} \leq \frac{1}{2}$ would lead to a contradiction with the minimality of a capacity vertex cover $x$. Hence $p_{m}>\frac{1}{2}$ follows.

Now, for any fixed $i=1,2, \ldots, m$, consider a component $F=(V(F), E(F))$ of the graph $\left(V, E^{x}\right)$ with $V(F) \subseteq I_{i} \cup H_{i}$. Assume, on contrary, that $\left(q_{i}, p_{i}\right) \neq$ $\Psi\left(\frac{\left|V(F) \cap H_{i}\right|}{\left|V(F) \cap I_{i}\right|}\right)$, or, in another words that the line

$$
\left\{(q, p): q\left|V(F) \cap I_{i}\right|+p\left|V(F) \cap H_{i}\right|=q_{i}\left|V(F) \cap I_{i}\right|+p_{i}\left|V(F) \cap H_{i}\right|\right\}
$$

is not tangent to $\partial T(1)$. But then, again, a small perturbation $(q, p)$ of $\left(q_{i}, p_{i}\right)$ on vertices $V(F)$, namely taking

$$
\left.x\right|_{V(F) \cap I_{i}}=q,\left.\quad x\right|_{V(F) \cap H_{i}}=\varphi(q)
$$


with properly chosen $q$ close to $q_{i}$ would decrease the value of the minimum capacitary vertex cover, a contradiction showing that for all such connected component $F$ the ratio $\frac{\left|V(F) \cap H_{i}\right|}{\left|V(F) \cap I_{i}\right|}$ is the same, as

$$
\left(q_{i}, p_{i}\right)=\Psi\left(\frac{\left|V(F) \cap H_{i}\right|}{\left|V(F) \cap I_{i}\right|}\right)=\Psi\left(\frac{\left|H_{i}\right|}{\left|I_{i}\right|}\right)
$$

then.

From this and $q_{1}<q_{2}<\cdots<q_{m}$ we easily conclude that

$$
\frac{\left|H_{1}\right|}{\left|I_{1}\right|}<\frac{\left|H_{2}\right|}{\left|I_{2}\right|}<\cdots<\frac{\left|H_{m}\right|}{\left|I_{m}\right|} .
$$

(b) Now we remove our additional assumptions that $K=\emptyset$ and that there are no edges within $H$. If we allow to add some edges within $H$ it cannot decrease that value $v c_{C}(G)$. But, as $\left.x\right|_{H}>\frac{1}{2}$, for each $u, v \in H(x(u), x(v)) \in T(1)$ automaticaly, so adding edges within $H$ will not increase $v c_{C}(G)$ either. If we allow $K \neq \emptyset$, we can extend $x$ from $I \cup H$ by taking $\left.x\right|_{K} \equiv \frac{1}{2}$. As $G[K]$ is Hallian, the minimum capacity vertex cover on this part will keep $x \equiv \frac{1}{2}$. As $\left.x\right|_{H}>\frac{1}{2}$, all constraints $(x(u), x(v)) \in T(1)$ for $u \in H$ and $v \in K$ will then be satisfied as well.

\section{The proof of Theorem 4}

Proof. By Theorem 3, if $\left.x\right|_{H}$ achieves $m \geq 1$ values $p_{1}>\cdots>p_{m}$ then corresponding sets satisfy $N\left(I_{1}\right)=H_{1}$,

$$
\frac{\left|H_{1}\right|}{\left|I_{1}\right|}<\frac{\left|H_{2}\right|}{\left|I_{2}\right|}<\frac{\left|H_{m}\right|}{\left|I_{m}\right|} .
$$

So, if $m \geq 2$ then

$$
\frac{\left|H_{1}\right|}{\left|I_{1}\right|}<\frac{|H|}{|I|}<\frac{\left|H_{m}\right|}{\left|I_{m}\right|} .
$$

easily follows, which contradicts our assumption that $I$ is a binding set; here $I_{1}$ provides the smaller ratio $\frac{\left|N\left(I_{1}\right)\right|}{\left|I_{1}\right|}$. Thus, under our assumptions we must have $m=1$, and $x$ is two-valued with $(q, p)=\Psi\left(\frac{|H|}{|I|}\right)$ by Theorem 3 . 